\title{
Construction of a new anti-CD19 chimeric antigen receptor and the anti-leukemia function study of the transduced $\mathrm{T}$ cells
}

\author{
Na An ${ }^{1}$, Zhongfei Tao ${ }^{1}$, Saisai Li ${ }^{1}$, Haiyan Xing ${ }^{1}$, Kejing Tang ${ }^{1}$, Zheng Tian ${ }^{1}$, Qing \\ Rao ${ }^{1}$, Min Wang ${ }^{1}$, Jianxiang Wang ${ }^{1}$ \\ ${ }^{1}$ State Key Laboratory of Experimental Hematology, Institute of Hematology and Blood Diseases Hospital, Chinese Academy \\ of Medical Sciences \& Peking Union Medical College, Tianjin, China \\ Correspondence to: Jianxiang Wang, e-mail: wangjx@ihcams.ac.cn \\ Min Wang, e-mail: wangjxm@ihcams.ac.cn \\ Keywords: chimeric antigen receptor, T cell, CD19, lentivirus, cellular immunotherapy \\ Received: October 01, $2015 \quad$ Accepted: January 23, $2016 \quad$ Published: January 30, 2016
}

\section{ABSTRACT}

Chimeric antigen receptor (CAR) transduced $T$ cells have been used to efficiently kill the target tumor cells depending on the single chain variable fragment (scFv) against the specific tumor associated antigen. Here we show the high specific cytotoxicity of the CAR-T cells with very low effector to target cell (E:T) ratio owing to the CD19-scFv, which was constructed in our laboratory and proved to be highly effective in our previous study. Four plasmids containing three generation of CAR were constructed by cloning the CD19-CAR fragment into the lentiviral vector PCDH. CD3 positive T cells were successfully transduced and the CAR protein expression was confirmed by flow cytometry and Western blot. When cocultured with CD19 positive leukemia cell line Nalm- 6 cells, CAR-T cells showed specific cytotoxicity: the percentage of target cells decreased to 0 in 24 hours; IL-2, IFN-Y and TNF-a produced in cocultured supernatants increased obviously; and the cytotoxicity reached more than $\mathbf{8 0} \%$, still remarkable even when the E:T ratio was as low as 1:4. Dynamic change of cell interaction between CAR-T and leukemia cells was visually tracked by using living cells workstation for the first time. A NOD/SCID B-ALL murine model was established using Nalm-6 cells inoculation with a morbidity rate of $100 \%$, and the survival time was prolonged statistically with CAR-T cell treatment. These data demonstrate that the CAR-T cells we prepared could be a promising treatment strategy for CD19 positive tumor diseases.

\section{INTRODUCTION}

Lymphocyte related diseases are very common in hematological malignancies. Acute lymphoblastic leukemia (ALL) occurs mostly in children. Although most of them can obtain complete remission and long term disease free survival through traditional chemotherapy, some still fail to achieve remission and progress to disease relapse [1-3]. The overall survival for adults with ALL is quite poor $[4,5]$. Allogeneic hematological stem cell transplantation (HSCT) is considered to be a curative therapy, but the rare matched donor and severe graft versus host disease (GVHD) hinder the general use of it.

Cellular immunotherapy is a new treatment strategy. The chimeric antigen receptor transduced $\mathrm{T}$ (CAR-T) cells have been considered to be a promising therapy recently [6-8]. CAR is a fusion protein connected by the following parts sequentially. The scFv is the light chain connected directly with the heavy chain of an antigen specific antibody responsible for recognizing the tumor specific antigen. The costimulatory molecules including CD28, OX40, CD137 and so on are important for $\mathrm{T}$ cell activation and proliferation. The $\mathrm{CD} 3 \zeta$ chain is the last part of signal transduction region to induce the final activation of CAR-T cells. The CAR is artificially classified into three generations depending on the number of costimulatory molecules it consists. The second generation consisting of only one or the third generation of two or more costimulatory molecules are used most widely. Once the scFv recognizes the specific antigen, CAR-T cell is activated and lyses the tumor cells. This is not MHC restricted and is highly antigen specific [9-11], with no risk of GVHD development as usually occurred in HSCT. 
CD19 is expressed on the surface of almost all B cell malignancies, but not on the hematopoietic stem cells and other tissue cells [12], so it has been an ideal target. Many centers have designed their own CD19-CAR-T cells proved to be effective and safe after testing in clinical trials [13-23].

Since the efficiency of CAR-T cells may be different due to the specific antigen recognition epitopes of $\mathrm{scFv}$, and we had constructed a highly effective CD19 specific scFv (CD19-scFv) in our previous study, which was used to construct our own CAR plasmids in this study. By preparing high quality lentivirus, CD3 positive $\mathrm{T}$ cells were successfully transduced and the function was confirmed using the CD28-CD3 $3-C A R-T$ cells as an example. In in vitro function study, we discovered that a very small amount of CAR-T cells were needed to lyse large number of target cells, which was different from most other reports requiring high E:T ratio. And we used living cells workstation for the first time to visually track cell interaction between CAR-T and leukemia cells. The xenograft mice model also showed anti-leukemic effect and safety in vivo.

\section{RESULTS}

\section{Four CD19-CAR lentiviral vectors were constructed}

We successfully constructed four kinds of CD19CAR lentiviral vectors using the CD19-scFv previously constructed in our laboratory. The one contained CD28 costimulatory domain named CD19scFv-CD8285 was used for the following function study. The sequences were combined in order of CD8 $\alpha$ leader, CD19-scFv, CD $8 \alpha$ hinge region, CD28 transmembrane and cytoplasmic domain and the $\mathrm{CD} 3 \zeta$ cytoplasmic region (Figure 1A). Part of the sequencing results were shown in Figure 1B.

\section{T cells were successfully transduced and expressed CAR protein}

CAR-T cells could express both GFP and CAR protein while empty vector transduced T (VEC-T) cells express GFP only. As shown in Figure 2A, cells were more than $95 \%$ of $\mathrm{CD}^{+}$after transduction and the $\mathrm{GFP}^{+} \mathrm{Fab}^{+}$ CAR-T cells were detected through flow cytometry. The transduction efficiency was about $50 \%$. So the $\mathrm{GFP}^{+}$cells could represent the $\mathrm{CAR}^{+}$cells. To further confirm the expression of CAR protein on $\mathrm{GFP}^{+}$cells, we sorted the $\mathrm{GFP}^{+} \mathrm{T}$ cells and performed the Western blot, the $\mathrm{GFP}^{+} \mathrm{T}$ cells of VEC-T cells were used as control. A $54 \mathrm{kD}$ fusion protein was detected in CAR-T cells (Figure 2B). We also study the proliferation efficiency of CAR-T cells by counting the number changes of CAR-T cells using trypan blue staining. The cells could proliferate more than 100 folds in 2 weeks under our culture system (Figure 2C).

\section{CAR-T cells showed specific cytotoxicity against CD19 $^{+}$leukemia cells}

In in vitro assay of the specific cytotoxicity of CD19CAR-T cells, we used CD19 ${ }^{+}$Nalm- 6 leukemia cells as target cells and CD19- U937 leukemia cells as control target cells. Compared to VEC-T cells, CAR-T cells showed obvious cytotoxicity against Nalm- 6 cells. As showed in Figure $3 \mathrm{~A}$, no matter the E:T ratio was as high as $6: 1$ or as low as $1: 3$, the $\mathrm{CD} 19^{+}$cells could not be detected by flow cytometry after 24 hours of coculture, but persisted in the control group even after 72 hours. And the flow charts were shown (Figure 3B). The difference of cells density was also observed under fluorescence microscope after 48 hours (Figure 3C), in which the red-colored cells represented residual Nalm-6 cells transfected with red fluorescent protein (RFP). Since the increase of cytokines concentration is the response of $\mathrm{T}$ cells activation and cytotoxicity, we detected the classic cytokines of IL- 2 , IFN- $\gamma$ and TNF- $\alpha$ as an example to evaluate the activation efficacy of CAR-T cells cocultured with target cells. The concentrations of IL-2, IFN- $\gamma$ and TNF- $\alpha$ were $(1186.34 \pm 15.5) \mathrm{pg} /$ $\mathrm{ml}, \quad(4943.93 \pm 29.46) \mathrm{pg} / \mathrm{ml}$ and $(899.345 \pm 15.72) \mathrm{pg} / \mathrm{ml}$ in the supernatant of Nalm6-CART coculture system, respectively, all were significantly higher than that of control groups $(P<0.001)$ (Figure 4A). CytoTox96 ${ }^{\circledR}$ NonRadioactive Cytotoxicity Assay based on the $\mathrm{LDH}$ release showed that even a small amount of CAR-T cells could kill a large number of Nalm- 6 cells. When E:T ratio was as low as 1:4, the cytotoxicity could be more than $60 \%$ and increased as the E:T ratio increase (Figure 4B). The above effects were not observed in targeting U937 cells. As showed in Figure 4C, the proportion of the Nalm-6 cells dropped dramatically in the coculture system, while that of the U937 cells kept raising in the coculture system over time. And the cytokines could not be detected in U937CART cells, which was significantly different from that of Nalm6-CART cells (Figure 4D).

\section{Target cells lysis were observed by microscopic imaging}

Nalm-6 cells transfected with RFP were cocultured with CAR-T cells or none transduced T (NTD-T) cells and immediately exposed to the microscopic imaging system. Videos tracking the cell interaction over time were made. Gradually disappeared red-colored cells representing Nalm-6 cells lysis were observed in the CAR-T group (Supplementary Video S1), but not in the NTD-T group (Supplementary Video S2). Red fluorescence intensity in CAR-T group decreased sharply in 8 hours but kept increasing in control group (Figure 5A). As shown in Figure 5B, dynamic binding rates in CAR-T group were remarkably higher than that of control group. Photographs of typical cell interaction including binding and then lysis were shown in Figure 5C and the corresponding video 
was shown in Supplementary Video S3. All of the above visually tracked study showed the direct binding and killing of target cells by CAR-T cells.

\section{CAR-T cells prolonged survival of B-ALL mice}

To evaluate the in vivo function of CAR-T cells, we established a B-ALL mouse model using Nalm- 6 cells inoculation. All transplanted mice developed aggressive acute lymphocytic leukemia with extensive infiltrations of $\mathrm{CD}_{1} 9^{+}$human cells in hematopoietic organs confirmed by flow cytometry and pathology (Figure 6A). The mean survival times of CAR-T cell treatment groups were prolonged significantly compared to that of control groups (Figure 6B). Mean survival times of Group A, $\mathrm{B}, \mathrm{C}$ and $\mathrm{D}$ were $(53.167 \pm 3.736) \mathrm{d},(47.000 \pm 1.000) \mathrm{d}$, $(43.833 \pm 1.195) \mathrm{d}$ and $(44.000 \pm 0.516) \mathrm{d}$, respectively. CAR-T treated Group A mice showed a longer survival time compared to all other groups $(P<0.05)$. Survival time of lower dose CAR-T treated Group B mice was also prolonged compared to that of Group $\mathrm{D}(P=0.009)$, but NTD-T cells could not prolong the survival time of Group $\mathrm{C}$ mice compared to that of Group D $(P=0.611)$. Thus demonstrated the anti-leukemia function of CAR-T cells in vivo and the efficiency could be improved when enough cells were used. No rapid body weight decrease (Figure $6 \mathrm{C}$ ) or other adverse effect were observed in all groups, indicating the safety of CAR-T cell treatment.

\section{DISCUSSION}

The cellular immune therapy has become a promising strategy in treatment of B cell malignancies. And the newly reported CAR-T cells have been proved to be incredible effective. The second generation CAR-T cells containing CD28 or CD137 costimulatory molecules are commonly used at present. Represented by the National Cancer Institute (NCI), Memorial SloanKettering Cancer Center (MSKCC) and so on, the clinical application of CD28-CAR-T cells is practicable. Although University of Pennsylvania (Upenn) center used the costimulatory molecule of CD137 in their CD137-CAR-T cells instead of CD28, there's no definite conclusion about which structure is better [24]. When CAR-T cells start to work, the first step is the specific recognition and binding of scFv to tumor antigen, which leads to the following CAR modified $T$ cell activation and cytotoxicity, so it's rational to consider that the difference in antigen recognition epitopes of $\mathrm{scFv}$ can also affect the function of CAR-T cells. The effect of CD19-scFv antibody against $\mathrm{B}$ lymphocyte malignancy has been confirmed in our previous study, we tried to investigate its function as a constituent part of the new CAR in the CAR-T cell application. Four CD19-CAR lentiviral vectors were successfully constructed. Here we focused only on CD28CAR-T cell function in vivo and in vitro, providing the basis for the following clinical application.

The function of CAR-T cells was comprehensively tested from three aspects, including the residual leukemia cells monitoring by flow cytometry, cytokines levels detecting by ELISA and cytotoxicity assay by LDH release. All results consistently showed strong cytotoxicity of the CD19-CAR-T cells against CD19 ${ }^{+}$leukemia cells. When E:T ratio was only 1:3, targeted leukemia cells could be cleared within 24 hours. Cytotoxicity could reach above $60 \%$ with an E:T ratio as low as 1:4. This was different from most other reports requiring more CAR-T cells, i.e. the higher E:T ratio. CD19- U937 leukemia cells were used as control target cells to confirm the specificity.

We visually track the dynamic change of CAR-T cells interacting with target cells, and the process of cell binding and lysis was clearly observed by using the living

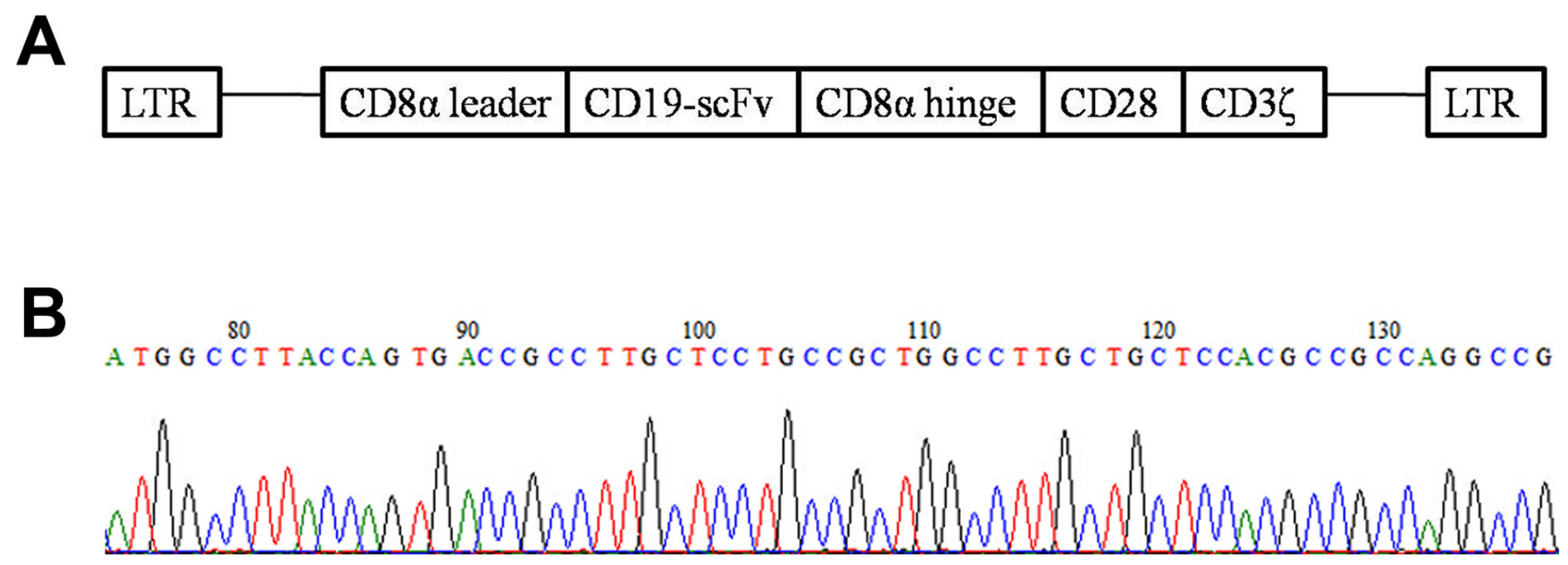

Figure 1: Structure diagram of CD19-CAR plasmid and part of the sequencing results. A. Structure diagram of the lentivial vector CD19scFv-CD828 . (LTR, long terminal repeat; CD8 $\alpha$ leader, sigal peptide of CD8 $\alpha$; scFv, single chain variable fragment; $\mathrm{CD} 8 \alpha$

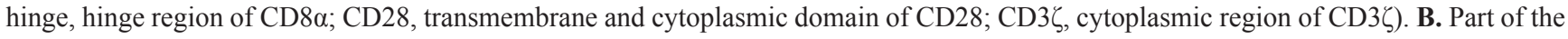
sequencing results, the first 21 amino acids of CD $8 \alpha$ leader. 

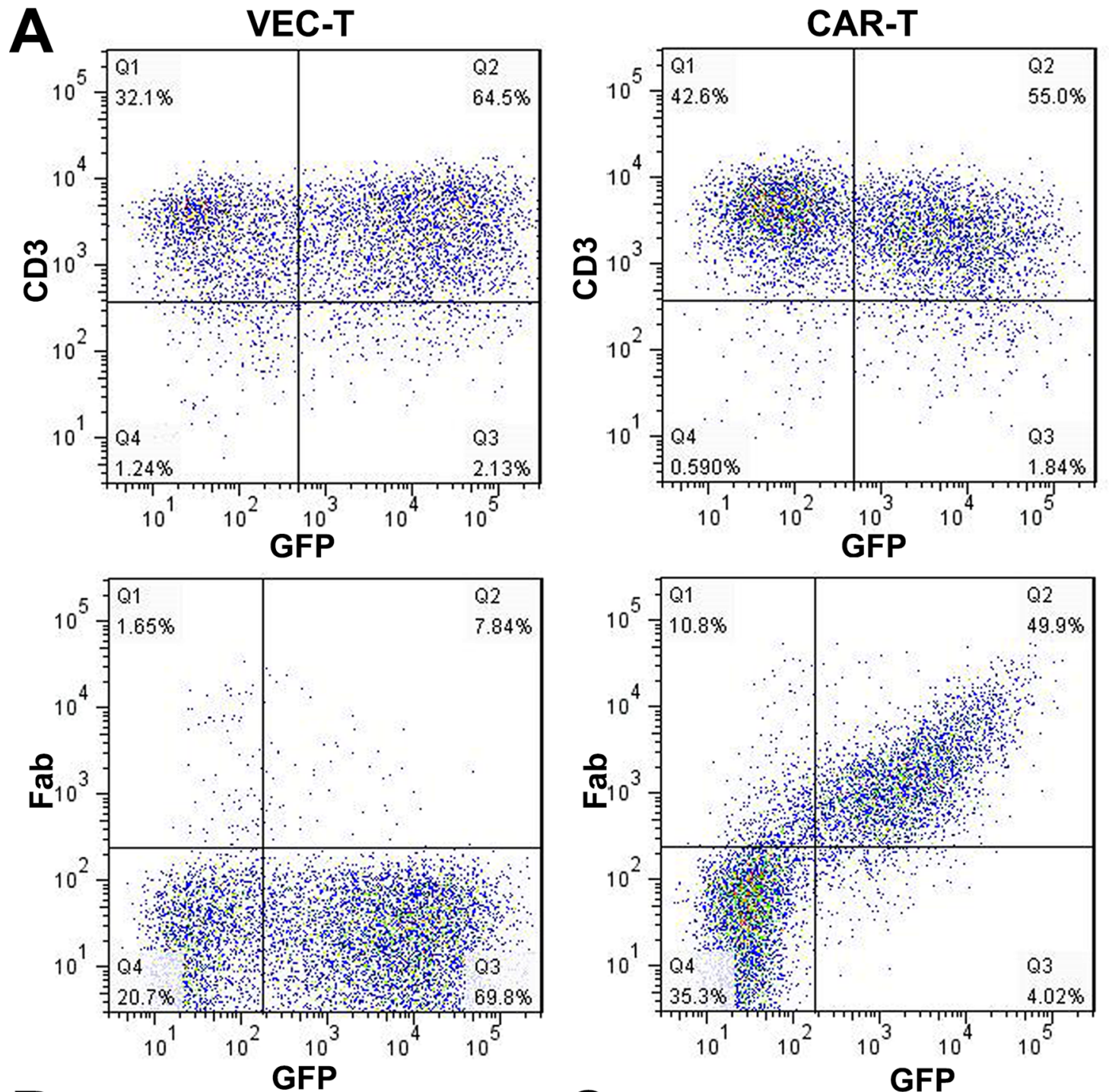

B

GFP
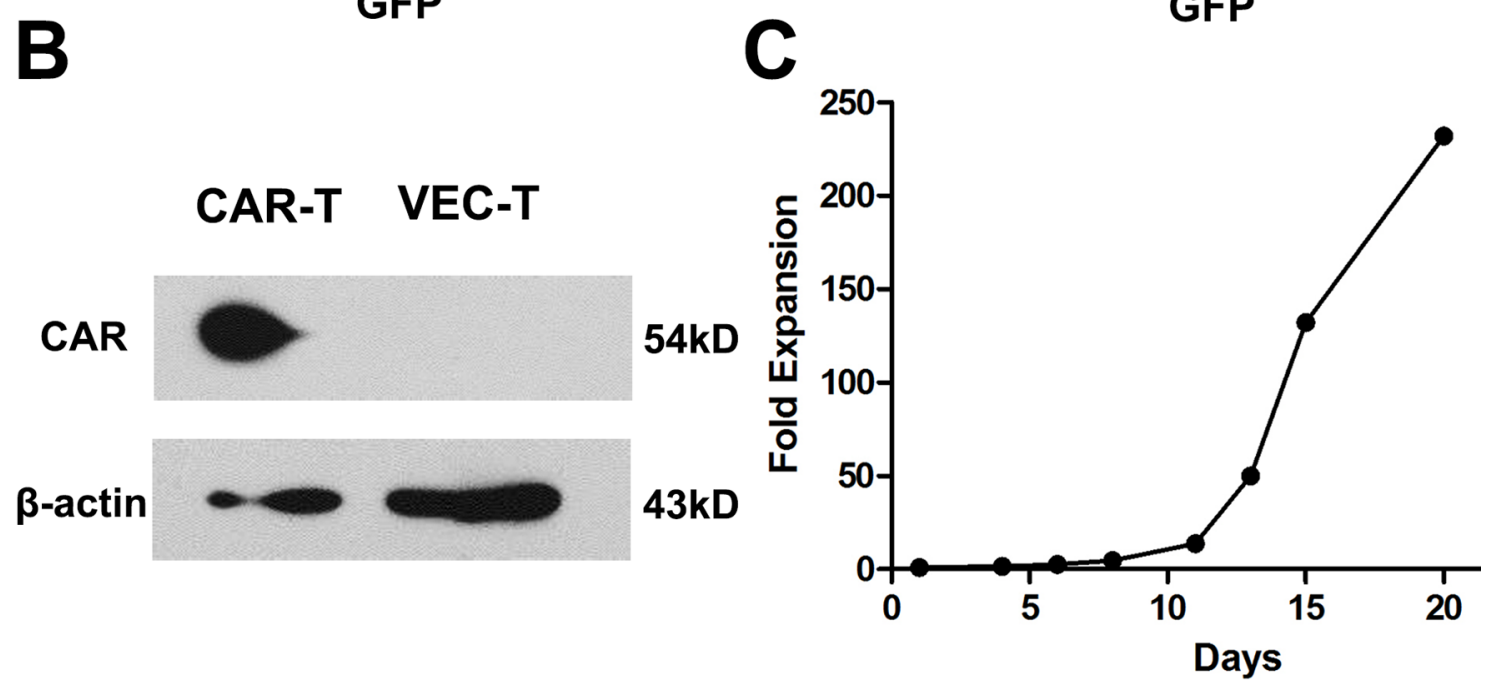

Figure 2: CAR protein expressed on transduced $T$ cells and the CAR-T cells proliferated efficiently. A. Flow cytometry to detect the CD3 and Fab expression on cell surface 4 days after virus transduction. CAR transduced cells (CAR-T, right panel) were more than $95 \%$ of $\mathrm{CD}^{+}$(right upper panel) and $50 \%$ of $\mathrm{GFP}^{+} \mathrm{Fab}^{+}$(right lower panel), while empty vector transduced cells (VEC-T, left panel)

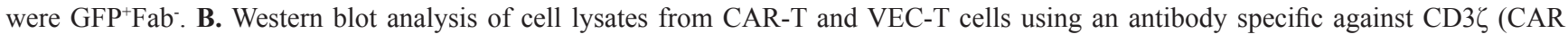
protein is $54 \mathrm{kD}$, while natural $\mathrm{CD} 3 \zeta$ is $16 \mathrm{kD}$ ). $\beta$-actin was used as a loading control. C. CAR-T cells were counted by trypan blue staining every 2 3 days after transduction. Fold expansion at each time point was calculated by dividing the cell number on transduction day. 

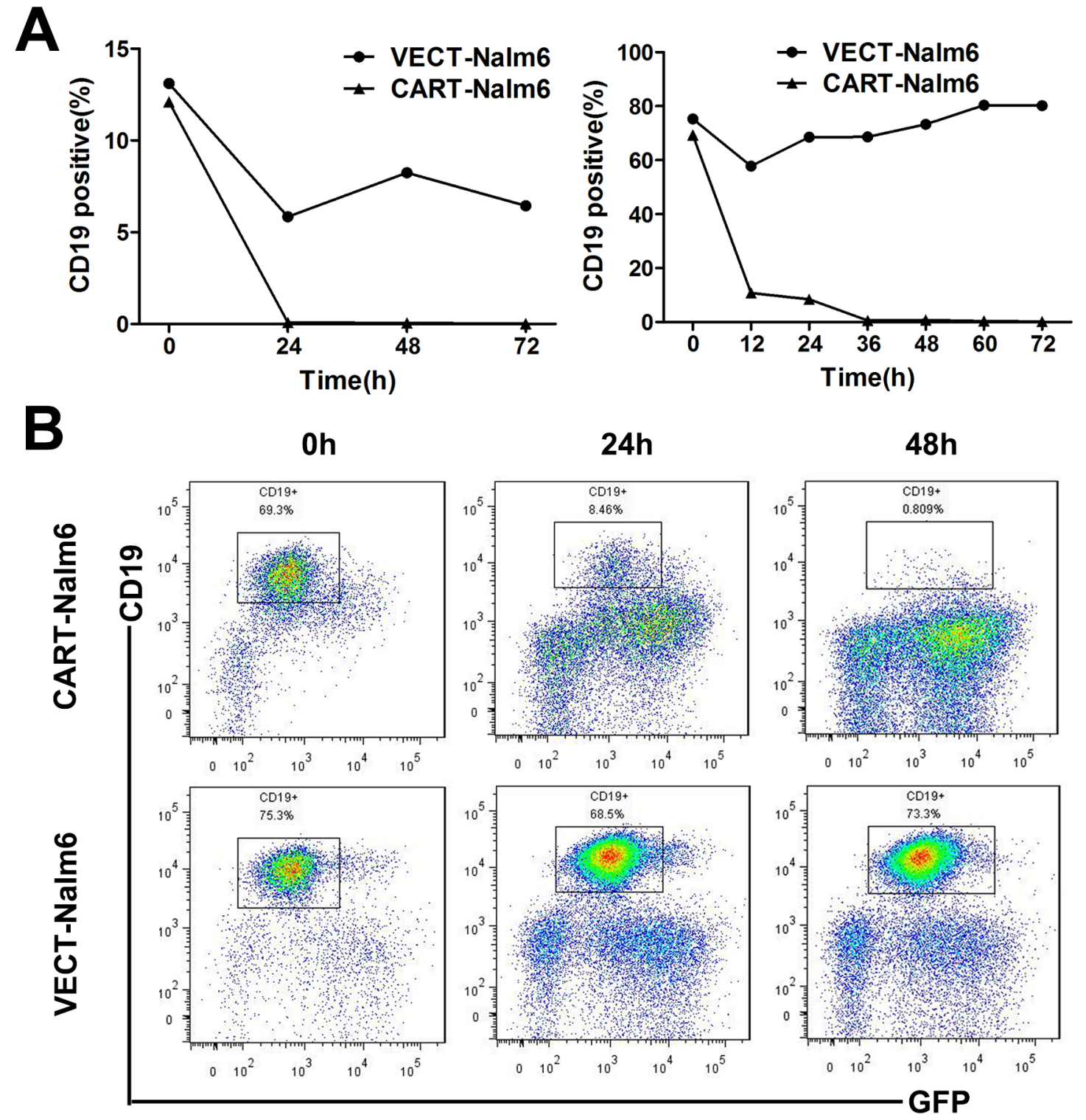

C

\section{CART-Nalm6}

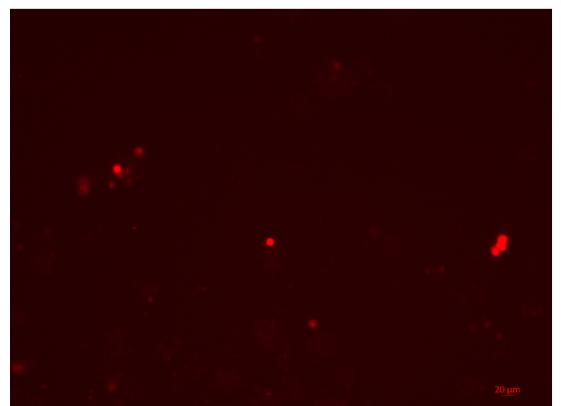

\section{VECT-Nalm6}

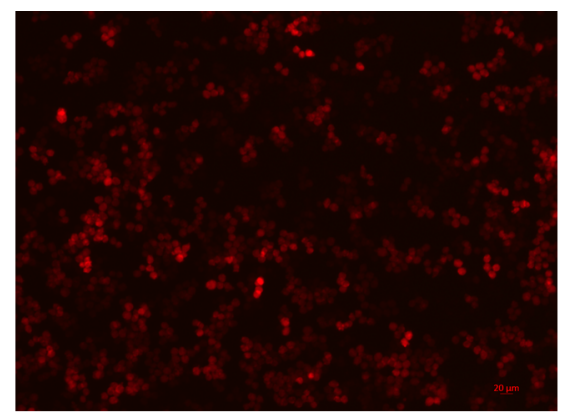

Figure 3: Nalm-6 leukemia cells could be eliminated by CAR-T cells. A. Percentage of CD19+ Nalm-6 cells detected by flow cytometry at different time points when cocultured with CAR-T cells or VEC-T cells as control. E:T ratio was 6:1 (left panel) or 1:3 (right panel). B. Diagram of flow cytometry showing the residual Nalm-6 cells when cocultured with CAR-T cells (upper panel) or VEC-T cells (lower panel) for 0h, 24h and 48h. C. Fluorescence microscopy of RFP ${ }^{+}$Nalm-6 cells after cocultured with CAR-T cells (left panel) or VEC-T cells (right panel) for 48 hours (magnification, $10 \times$ ). 

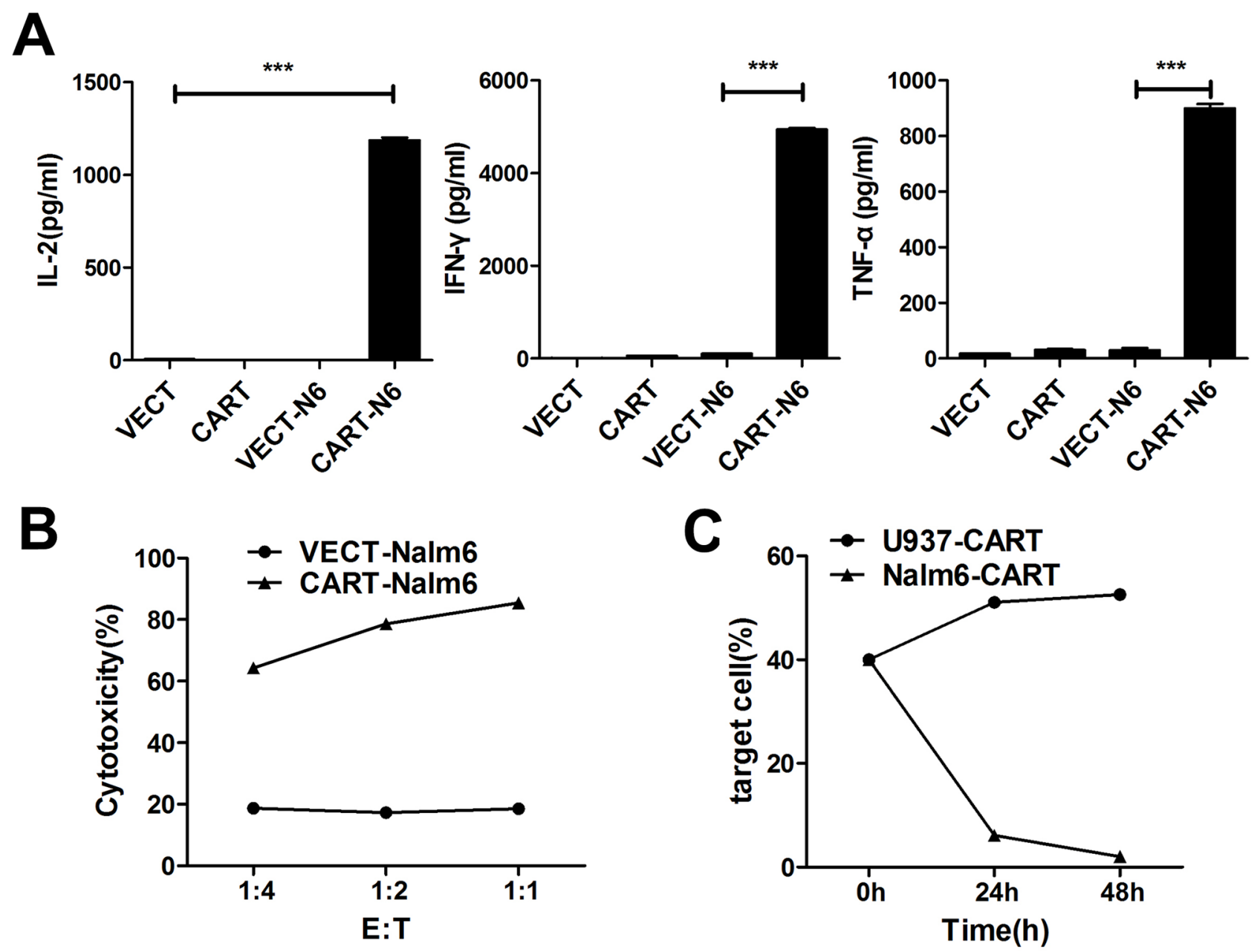

D
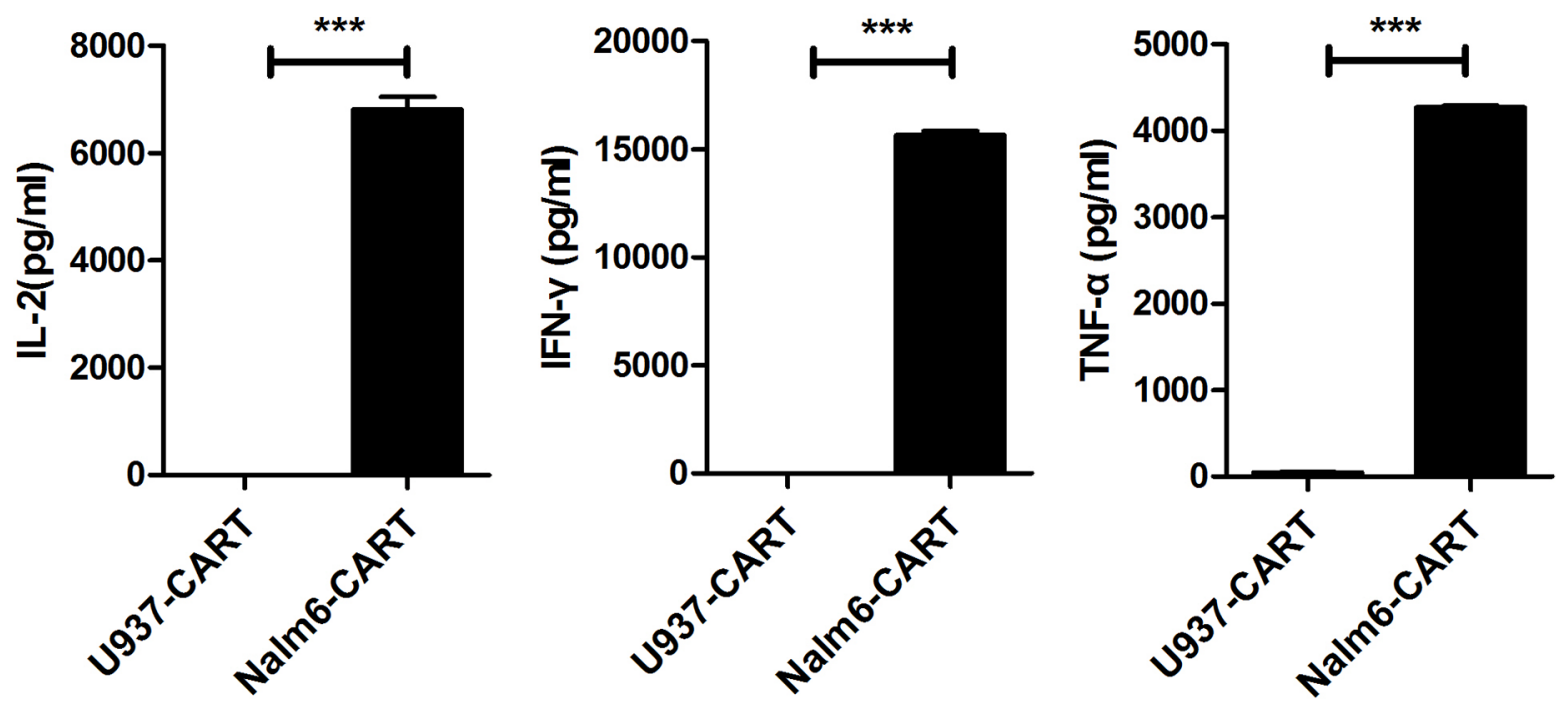

Figure 4: CAR-T cells showed specific cytotoxicity against CD19 ${ }^{+}$Nalm-6 cells but no effect on CD19- U937 cells. A. ELISA detection of IL-2, IFN- $\gamma$ and TNF- $\alpha$ in the cultured supernatants of VECT (VEC-T), CART (CAR-T), VECT-N6 (VEC-T cocultured with Nalm-6) and CART-N6 (CAR-T cocultured with Nalm-6) cultured for 24 hours. Results are shown as mean \pm SD from three independent experiments. B. CytoTox $96^{\circledR}$ Non-Radioactive Cytotoxicity Assay of the cell cytotoxicity at different E:T ratios. The cytolytic activity was measured by assays of lactate dehydrogenase $(\mathrm{LDH})$ release after cocultured for $7 \mathrm{~h}$ using the detection kit according to the manufacturer's instructions. Cytotoxicity is reported as the mean \pm SD of triplicate determinations. C. Flow cytometry detection of the percentage of target cells (U937 or Nalm-6) cocultured with CAR-T cells at different time points. D. ELISA detection of different cytokines in U937-CART (U937 and CAR-T) or Nalm6-CART (Nalm-6 and CAR-T) coculture system after 24 hours. Results are shown as mean \pm SD from three independent experiments. 


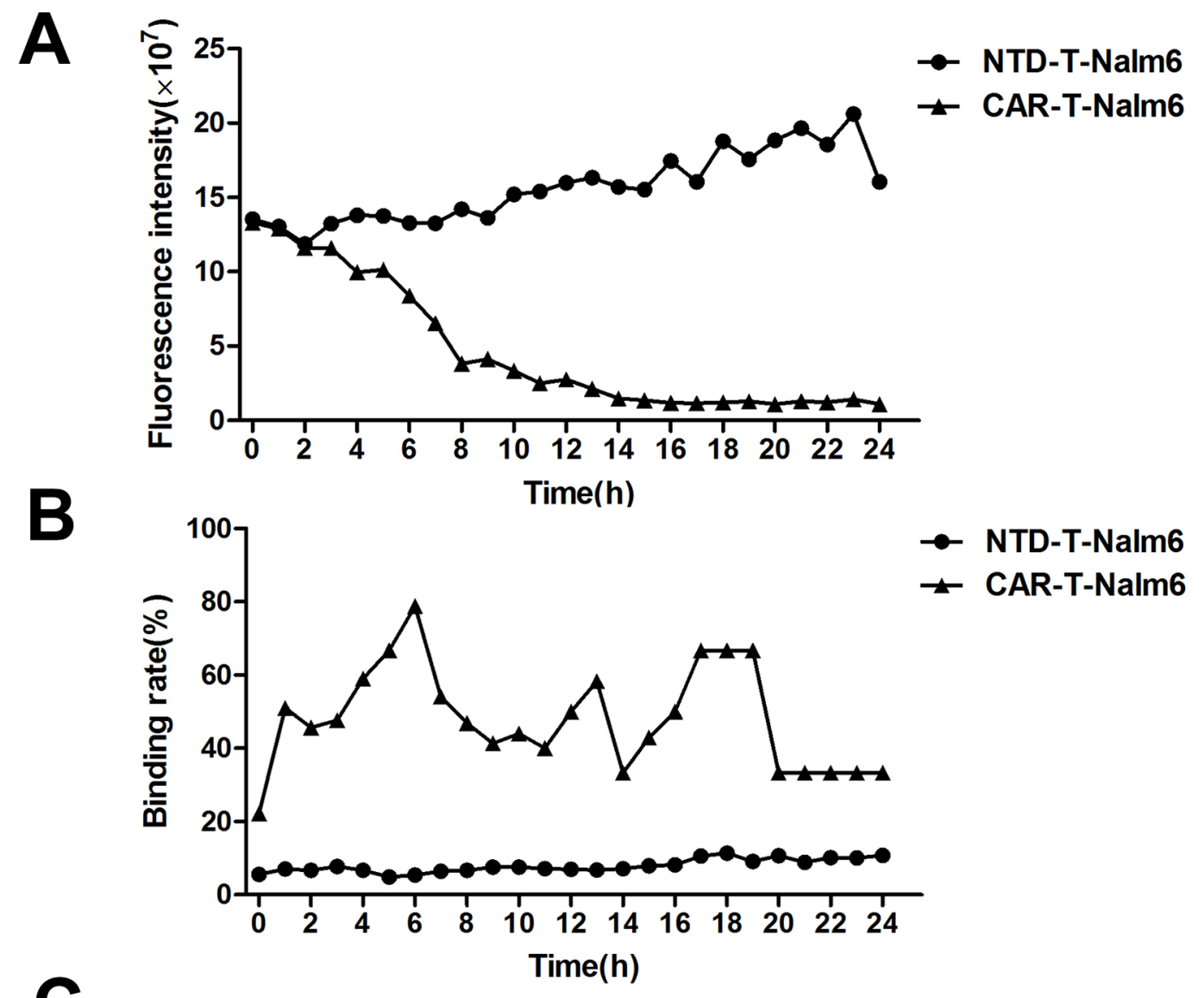

Oh

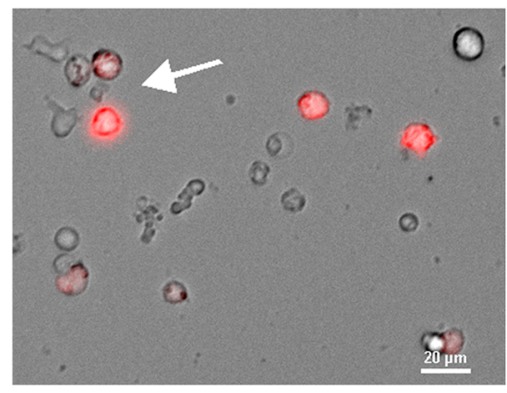

$9 \mathrm{~h}$

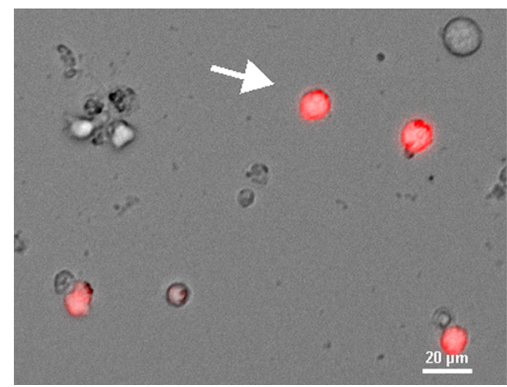

$1 \mathrm{~h}$

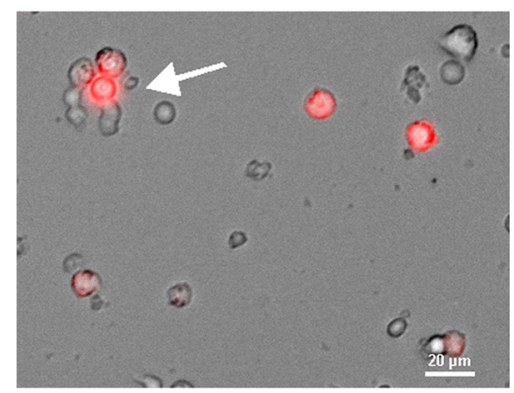

$10 \mathrm{~h}$

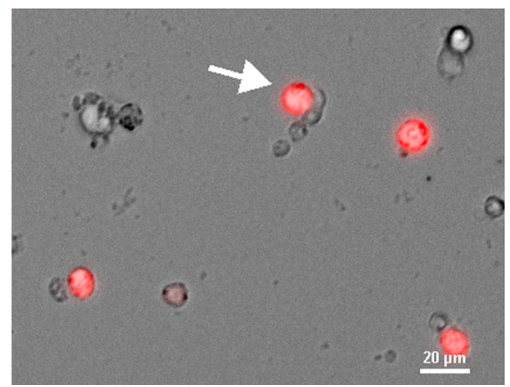

9h

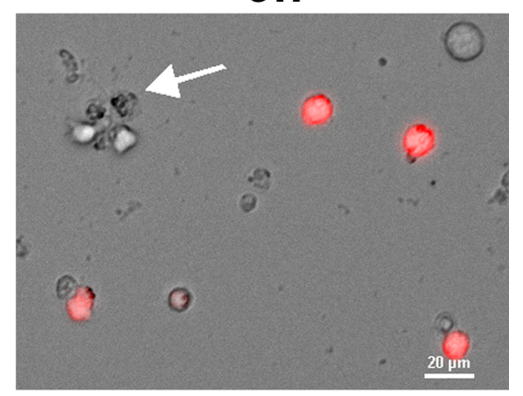

$18 \mathrm{~h}$

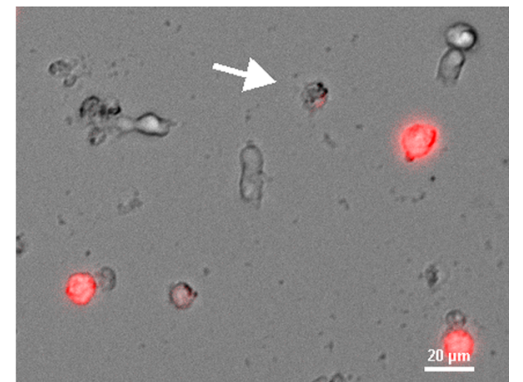

Figure 5: Dynamic observation of cell interaction by using living cells workstation. A. Fluorescence intensity of RFP ${ }^{+} \mathrm{Nalm}^{-}$ 6 cells changes over time analyzed by Elements 3.1 and Velocity 6.1 software. CAR-T-Nalm6 represents Nalm- 6 cells cocultured with CAR-T cells, while NTD-T-Nalm6 represents Nalm-6 cells cocultured with none transduced T cells (NTD-T). B. Binding rates of Nalm-6 cells to CAR-T cells or NTD-T cells at different time points were analyzed by Elements 3.1 and Velocity 6.1 software. C. Photographs representing the process of cell binding and lysis (arrows) captured by living cells workstation (Nikon Ti-e). Red-colored cells were RFP ${ }^{+}$ Nalm- 6 cells and others were CAR-T cells. 
A
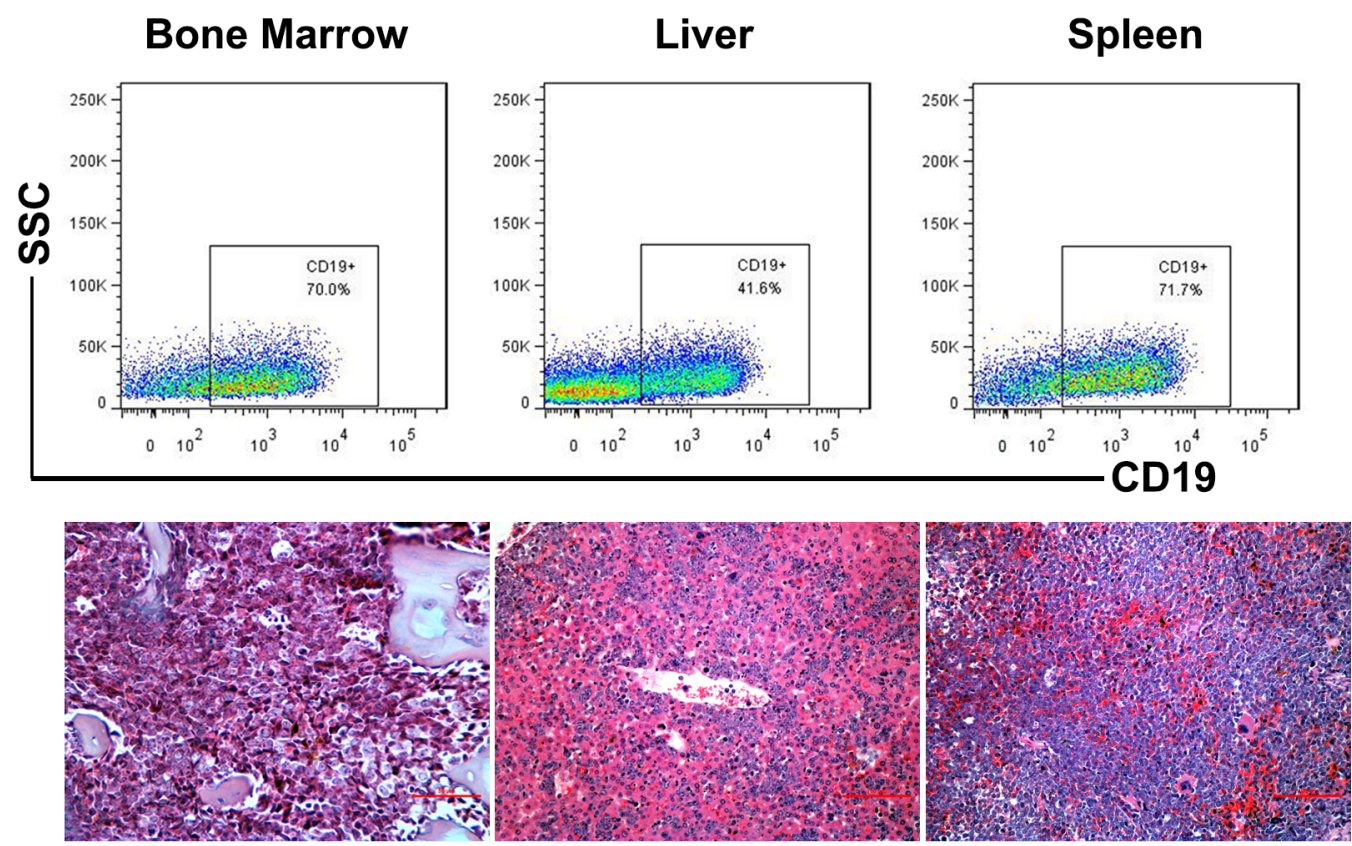

B
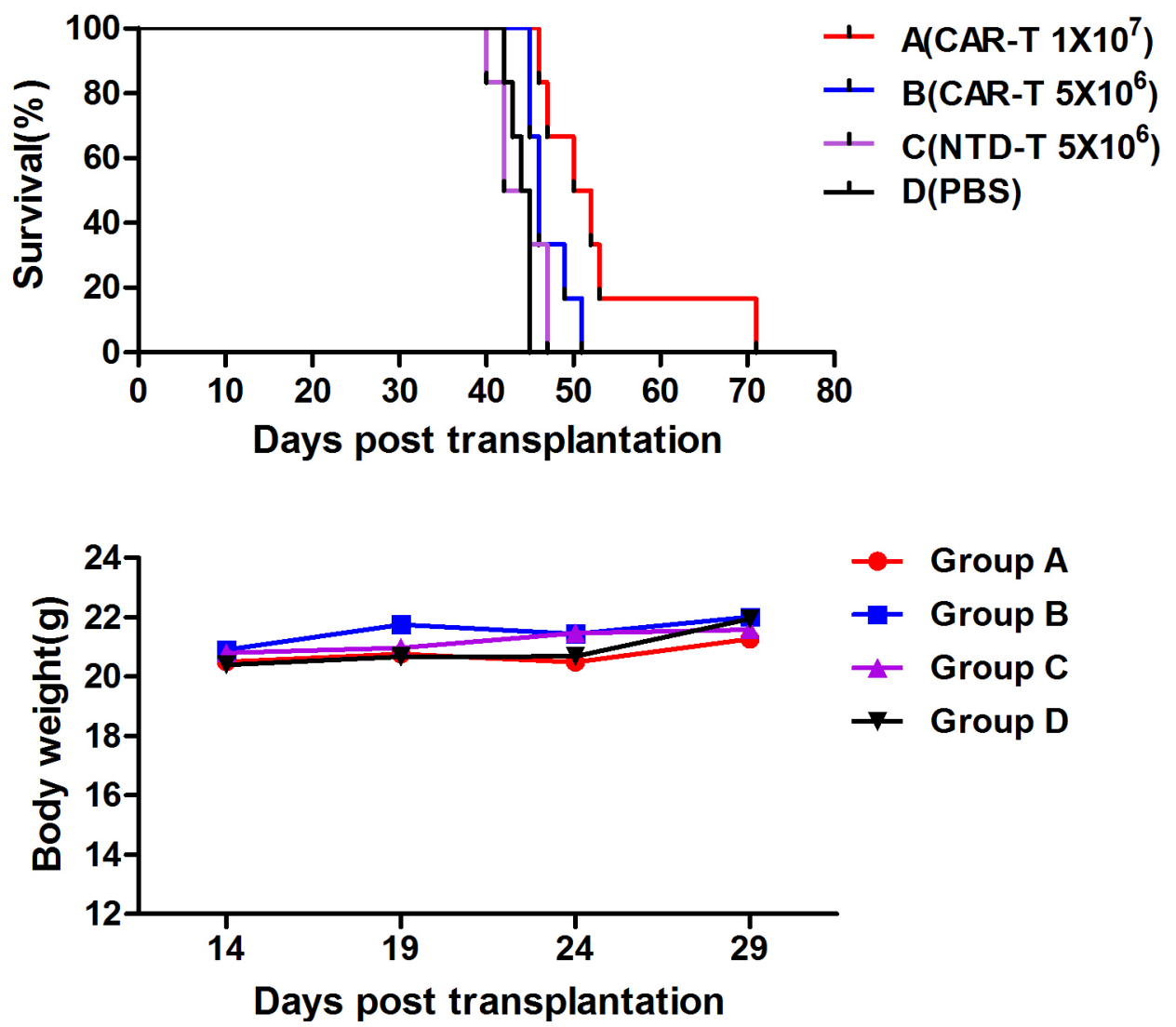

Figure 6: In vivo CAR-T cell treatment in murine B-ALL model. A. Detection of human CD19+ cells by flow cytometry (upper panel) and histopathologic analysis (lower panel) of bone marrow, liver and spleen from Nalm-6 cells inoculated mice. B. Kaplan-Meier survival curves of four treatment groups. The $P$-values were determined by log-rank test. $P<0.05$ when group A compared with all other groups. $P=0.009$ when group $\mathrm{B}$ compared with group $\mathrm{D}$, but no difference between group $\mathrm{C}$ and $\mathrm{D}(P=0.611)$. C. Average body weight of four groups $(\mathrm{n}=6)$ after CAR-T cell treatment. CAR-T cells were administered at day 15 after Nalm-6 cells inoculation. 
cells workstation for the first time. To further evaluate the effect of CAR-T cells in vivo, we successfully established a B-ALL murine model with a morbidity of $100 \%$. CAR-T cell treatment extended the survival of mice compared with that of control groups, the efficacy was more obvious with a higher dosage of CAR-T cells. The mice did not achieve long term disease-free survival, possibly due to the failure of CD28-CAR-T cells to proliferate persistently in vivo. But the effect could be improved by increasing cell numbers and infusion frequencies of CAR-T cells, and at the same time to avoid the risk of persistent B cells aplasia. Function of CAR-T cells are influenced by many factors, including the CAR structure ( $\mathrm{scFv}$ and costimulatory molecules, etc), culture condition in vitro and T cell subtype [25-29], infusion time, dose and frequency, and usage of other drugs (chemotherapy, IL-2 and PD-1 inhibitors, etc). Therefore, we will optimize various conditions to gain the best in vivo treatment effect in future.

In conclusion, we successfully constructed four new CD19-CAR lentiviral vectors and transduced T cells. The CAR-T cells showed strong specific cytotoxicity against CD19+ leukemia cells in comprehensive function study in vitro. Preliminary animal experiments also indicated its anti-leukemia effect in vivo, and further study is needed to optimize the therapeutic efficacy.

\section{MATERIALS AND METHODS}

\section{Construction of anti-CD19 CAR lentiviral vectors}

The mouse anti-human CD19-scFv segment was cloned from the PET22b plasmid containing the $\mathrm{V}_{\mathrm{L}^{-}}$ linker- $\mathrm{V}_{\mathrm{H}}$ sequence generated by our laboratory before. The $\mathrm{scFv}$ sequence optimized suitable for expression in human cells was linked to $\mathrm{CD} 8 \alpha$ signal sequence with NheI and EcoRI sites at both ends. The sequences of CD8 $\alpha$ hinge and transmembrane region, the CD28 transmembrane and cytoplasmic domain and the $\mathrm{CD} 137$ or $\mathrm{CD} 3 \zeta$ cytoplasmic region were all referred to the NCBI GeneBank based on other reports. The above signal transduction regions were all amplified from the cDNA of a healthy donor's peripheral blood mononuclear cells (PBMC) by PCR and connected in order by overlap PCR. And EcoRI and NotI sites were included. The two parts were finally connected by enzyme digestion and ligation, and the entire sequence was cloned into the lentiviral vector pCDH (System Bioscience, USA) with GFP marker gene, named pCDH-CAR plasmid. All sequences were confirmed by gene sequencing (Life Technologies, China). The empty vector $\mathrm{pCDH}$ was used as control vector.

\section{Lentivirus production}

To produce lentiviruses, $293 \mathrm{~T}$ packaging cells were transfected with empty vector or pCDH-CAR plasmids as mentioned above. The packaging plasmids were psPAX2 and pMD2.G (Invitrogen, USA). The day before transfection, $293 \mathrm{~T}$ cells were plated in the $10 \mathrm{~cm}$ dish cultured in DMEM (Invitrogen, USA) with 10\% FBS (Hyclone, USA). When cell density reached 60\% 80\%, the cells were transfected with the above plasmids using polyethylenimine (PEI, Polysciences, USA). After the transfected cells incubated in $37^{\circ} \mathrm{C}, 5 \% \mathrm{CO}_{2}$ incubator for $12 \sim 16$ hours, the culture medium was replaced with $5 \sim 6 \mathrm{ml}$ fresh DMEM with $10 \%$ FBS. The supernatants containing viruses were harvested at 24 hours and 48 hours and concentrated by ultracentrifugation for 90 minutes at $50,000 \mathrm{~g}, 4^{\circ} \mathrm{C}$, then stored at $-80^{\circ} \mathrm{C}$ until use.

\section{$T$ cells isolation and transduction}

Peripheral blood of healthy donors was obtained from Tianjin Blood Center. CD3 positive T cells were isolated and enriched by CD3 enrichment antibody cocktail (Stem Cell, USA) and Ficoll solution (TBD Science, China). The cell purity was detected by flow cytometry (CantoII, BD, USA) stained with APC-labeled mouse anti-human CD3 antibody (Biolegend, USA). T cells maintained in RPMI 1640 (Invitrogen, USA) plus $10 \%$ FBS were inoculated in 24-well plates embedded with CD3 and CD28 antibody (eBioscience, USA) with a cell density of $1 \sim 1.5 \times 10^{6} / \mathrm{ml}$, and rhIL-2 (R\&D, USA) was added with a dose of $200 \mathrm{U} / \mathrm{ml}$. After 24 hours, cells were transduced with thawed lentiviruses which were added directly to the plate. $8 \mu \mathrm{g} / \mathrm{ml}$ polybrene (Sigma, USA) was added. The plate was then centrifuged for 90 minutes at $1800 \mathrm{rpm}, 32^{\circ} \mathrm{C}$ and incubated for another 24 hours without disturbing. The other day, the transduction was repeated once. Then culture medium was changed every 2 days and cells were kept in flasks with a density of $1.0 \times 10^{6} / \mathrm{ml}$ with $\mathrm{rhIL}-2$ routinely used.

\section{CAR protein detection on transduced cells}

Flow cytometry and Western blot analysis were done to confirm the expression of CAR protein. In flow cytometry, transduced cells were harvested and washed once with PBS, stained with biotin-labeled goat antimouse IgG, F(ab') 2 antibodies (Jackson Immunoresearch, USA) for 20 minutes at $4^{\circ} \mathrm{C}$ and washed twice. Then APC/Cy7-conjugated streptavidin antibodies (Biolegend, USA) were added, incubated at $4^{\circ} \mathrm{C}$ for another 20 minutes and washed once. Then the cells were analyzed by flow cytometry and results were analyzed with FlowJo software. In Western blot analysis, GFP positive cells were sorted by BD FACS AriaIII System and lysed with buffer containing $50 \mathrm{mMTris} / \mathrm{HCl}(\mathrm{pH} 7.5), 150 \mathrm{mMNaCl}, 1 \%$ Triton X-100, 1\% sodium deoxycholate, $0.1 \%$ SDS and $0.1 \mathrm{mM}$ PMSF. Proteins were analyzed by SDS-PAGE and Western blotting. Membranes were incubated with primary antibodies against human $\mathrm{CD} 3 \zeta$ (BD Pharmingen, USA) and $\beta$-actin (Cell Signaling Technology, USA). Blots were 
visualized using the ECL (Millipore, USA) with Image Quant LAS-4010 (GE Healthcare, USA). The empty vector transduced cells were used as control.

\section{In vitro function study of CAR-T cells}

In order to verify the specific cytotoxicity of CAR-T cells against CD19 ${ }^{+}$leukemia cells, a B-cell acute lymphoblastic leukemia cell line Nalm-6 cells were used as the target cell. VEC-T cells were used as control effector cells. Effector and target cells were cocultured in 24-well plates with an E:T ratio of $6: 1$ or $1: 3$. Repetitive wells were set for detection at different time points $(0 \mathrm{~h}, 12 \mathrm{~h}, 24 \mathrm{~h}, 36 \mathrm{~h}, 48 \mathrm{~h}, 64 \mathrm{~h}$ and $72 \mathrm{~h}$ ). Each time point, cells were harvested and washed once, stained with $\mathrm{PE} / \mathrm{Cy} 7$-conjugated mouse anti-human CD19 antibody (Biolegend, USA) for 20 minutes on ice, then washed and resuspended in PBS for flow cytometry analysis. The percentage of $\mathrm{CD} 19^{+}$ cells represented the residual level of target cells. The supernatants were harvested and stored as aliquots at $-20^{\circ} \mathrm{C}$ for the assay of cytokines produced in the coculture system, including IL-2, IFN- $\gamma$ and TNF- $\alpha$ using ELISA kits (R\&D, USA). The supernatants from CAR-T cells or VEC-T cells cultured alone were also harvested and used as control groups to exclude the natural cytokine release. Absorbance was measured with Synergy H4 Hybrid Microplate Reader (Biotek, USA) at $570 \mathrm{~nm}$. CytoTox96 ${ }^{\circledR}$ Non-Radioactive Cytotoxicity Assay (Promega, USA) was further used to assess the specific lysis of target cells according to the manufacture's protocol with the E:T ratio of $1: 4$, $1: 2$ and 1:1. Three repeated wells were designed. After 7 hours, the OD value was detected at $490 \mathrm{~nm}$ and cytotoxicity was calculated according to the formula. In order to verify the specific cytotoxicity of CAR-T cells, acute myelomonocytic leukemia cell line U937 cells were used as control target cells in above experiments.

\section{Dynamic imaging of cell interaction by living cells workstation}

To monitor the dynamic change of cells in the coculture system, RFP transfected Nalm-6 cells were mixed with CAR-T cells or NTD-T cells at 1:1, respectively. A total volume of $400 \mu$ cell mixture was inoculated in a 24-well plate for 1 minute at room temperature. Then bright field and TRITC red channel video was captured by the living cells workstation (Nikon Ti-e, Japan) for 24 hours. The exposure time was $5 \mathrm{~s}$ and pictures were taken every 10 minutes under $37^{\circ} \mathrm{C}$, $5 \% \mathrm{CO}_{2}$. RFP fluorescence intensity and cell binding rate changes over time were analyzed by Elements 3.1 and Velocity 6.1 software. The percentage of Nalm- 6 cells bound to $T$ cells in all Nalm- 6 cells in one field was defined as binding rate.

\section{Establishment of murine B-ALL model and in vivo treatment with $\mathrm{CAR}-\mathrm{T}$ cells}

To establish the B-ALL mouse model, 4 weeks old NOD/SCID female mice were purchased from Institute of Laboratory Animal Sciences (CAMS\&PUMC, China). After adaptive feeding for 2 weeks, 24 mice were irradiated at $200 \mathrm{cGy}$ and intravenously inoculated with $5 \times 10^{5} \mathrm{Nalm}-6$ cells. Two weeks after transplantation, $1 \%$ $\mathrm{CD} 19^{+}$human cells were detected in peripheral blood by flow cytometry. Then the mice were randomized into four treatment groups. Group A were injected intravenously with $1 \times 10^{7}$ CAR-T cells, Group B with $5 \times 10^{6}$ CAR-T cells, Group C with $5 \times 10^{6}$ NTD-T cells and Group $\mathrm{D}$ with the equivalent volume of PBS. The disease development was assessed by percentages of circulating CD $19^{+}$leukemia cells by flow cytometry. Symptoms including diarrhea, paralysis and body weight loss were also monitored. The overall survival was measured from the date of transplantation until death. Dead mice were dissected for pathological and flow cytometry analysis to confirm the diagnosis of leukemia. All animal experiments were approved by the Institutional Animal Care and Use Committee of Peking Union Medical College.

\section{Statistical analysis}

SPSS software (version 16.0) was used for statistical analysis. The comparisons were performed by Student's $t$-test analysis using GraphPad Prism (version 5.0). The lifespan of mice was analyzed by Kaplan-Meier methods and a log-rank test. $P$-values $<0.05$ were considered statistically significant. Briefly, ${ }^{*} P<0.05, * * P<0.01$, and $* * * P<0.001$ in comparison.

\section{ACKNOWLEDGMENTS}

We are grateful to kind assistance from the staffs (especially Wanzhu Yang) in Core Facility of flow cytometry and living cells workstation, State Key Laboratory of Experimental Hematology, Institute of Hematology and Blood Diseases Hospital, Chinese Academy of Medical Sciences and Peking Union Medical College. This work was supported by National Natural Science Foundation of China $(81430004,81370633)$ and Foundation for Innovative Research Groups of the National Natural Science Foundation of China (81421002).

\section{CONFLICTS OF INTEREST}

The authors declare no conflicts of interest exist. 


\section{Author contributions}

N.A performed all the experiments, analyzed the data and wrote the manuscript. Z.T and S.L offered important advices and helped with some experiments. H.X helped with the mice experiments. Z.T and K.T helped in blood samples preparation. Q.R, M.W and J.W conceived, designed and supervised the study, reviewed and approved the manuscript.

\section{REFERENCES}

1. Bhojwani D and Pui CH. Relapsed childhood acute lymphoblastic leukaemia. Lancet Oncol. 2013; 14: e205-217.

2. Nguyen K, Devidas M, Cheng SC, La M, Raetz EA, Carroll WL, Winick NJ, Hunger SP, Gaynon PS and Loh ML. Factors influencing survival after relapse from acute lymphoblastic leukemia: a Children's Oncology Group study. Leukemia. 2008; 22: 2142-2150.

3. Raetz EA and Bhatla T. Where do we stand in the treatment of relapsed acute lymphoblastic leukemia? Hematology Am Soc Hematol Educ Program. 2012; 2012: 129-136.

4. Pulte D, Redaniel MT, Jansen L, Brenner H and Jeffreys M. Recent trends in survival of adult patients with acute leukemia: overall improvements, but persistent and partly increasing disparity in survival of patients from minority groups. Haematologica. 2013; 98: 222-229.

5. Pulte D, Jansen L, Gondos A, Katalinic A, Barnes B, Ressing M, Holleczek B, Eberle A and Brenner H. Survival of adults with acute lymphoblastic leukemia in Germany and the United States. PLoS One. 2014; 9: e85554.

6. Barrett DM, Singh N, Porter DL, Grupp SA and June CH. Chimeric antigen receptor therapy for cancer. Annu Rev Med. 2014; 65: 333-347.

7. Maude SL, Teachey DT, Porter DL and Grupp SA. CD19targeted chimeric antigen receptor T-cell therapy for acute lymphoblastic leukemia. Blood. 2015; 125: 4017-4023.

8. Maus MV, Grupp SA, Porter DL and June CH. Antibodymodified T cells: CARs take the front seat for hematologic malignancies. Blood. 2014; 123: 2625-2635.

9. Gross G, Waks T and Eshhar Z. Expression of immunoglobulin-T-cell receptor chimeric molecules as functional receptors with antibody-type specificity. Proc Natl Acad Sci U S A. 1989; 86: 10024-10028.

10. Eshhar Z, Waks T, Gross G and Schindler DG. Specific activation and targeting of cytotoxic lymphocytes through chimeric single chains consisting of antibody-binding domains and the gamma or zeta subunits of the immunoglobulin and T-cell receptors. Proc Natl Acad Sci U S A. 1993; 90: 720-724.

11. Satta A, Mezzanzanica D, Turatti F, Canevari S and Figini M. Redirection of T-cell effector functions for cancer therapy: bispecific antibodies and chimeric antigen receptors. Future Oncol. 2013; 9: 527-539.
12. Scheuermann RH and Racila E. CD19 antigen in leukemia and lymphoma diagnosis and immunotherapy. Leuk Lymphoma. 1995; 18: 385-397.

13. Brentjens RJ, Riviere I, Park JH, Davila ML, Wang X, Stefanski J, Taylor C, Yeh R, Bartido S, Borquez-Ojeda O, Olszewska M, Bernal Y, Pegram H, et al. Safety and persistence of adoptively transferred autologous CD19-targeted $\mathrm{T}$ cells in patients with relapsed or chemotherapy refractory B-cell leukemias. Blood. 2011; 118: 4817-4828.

14. Davila ML, Riviere I, Wang X, Bartido S, Park J, Curran K, Chung SS, Stefanski J, Borquez-Ojeda O, Olszewska M, Qu J, Wasielewska T, He Q, et al. Efficacy and toxicity management of 19-28z CAR T cell therapy in B cell acute lymphoblastic leukemia. Sci Transl Med. 2014; 6: 224ra225.

15. Maude SL, Frey N, Shaw PA, Aplenc R, Barrett DM, Bunin NJ, Chew A, Gonzalez VE, Zheng Z, Lacey SF, Mahnke YD, Melenhorst JJ, Rheingold SR, et al. Chimeric antigen receptor $\mathrm{T}$ cells for sustained remissions in leukemia. $\mathrm{N}$ Engl J Med. 2014; 371: 1507-1517.

16. Lee DW, Kochenderfer JN, Stetler-Stevenson M, Cui YK, Delbrook C, Feldman SA, Fry TJ, Orentas R, Sabatino M, Shah NN, Steinberg SM, Stroncek D, Tschernia N, et al. $\mathrm{T}$ cells expressing CD19 chimeric antigen receptors for acute lymphoblastic leukaemia in children and young adults: a phase 1 dose-escalation trial. Lancet. 2015; 385: 517-528.

17. Kalos M, Levine BL, Porter DL, Katz S, Grupp SA, Bagg A and June $\mathrm{CH}$. T cells with chimeric antigen receptors have potent antitumor effects and can establish memory in patients with advanced leukemia. Sci Transl Med. 2011;3: 95ra73.

18. Brentjens RJ, Davila ML, Riviere I, Park J, Wang X, Cowell LG, Bartido S, Stefanski J, Taylor C, Olszewska M, Borquez-Ojeda O, Qu J, Wasielewska T, et al. CD19targeted $\mathrm{T}$ cells rapidly induce molecular remissions in adults with chemotherapy-refractory acute lymphoblastic leukemia. Sci Transl Med. 2013; 5: 177ra138.

19. Grupp SA, Kalos M, Barrett D, Aplenc R, Porter DL, Rheingold SR, Teachey DT, Chew A, Hauck B, Wright JF, Milone MC, Levine BL and June $\mathrm{CH}$. Chimeric antigen receptor-modified T cells for acute lymphoid leukemia. $\mathrm{N}$ Engl J Med. 2013; 368: 1509-1518.

20. Porter DL, Levine BL, Kalos M, Bagg A and June CH. Chimeric antigen receptor-modified $\mathrm{T}$ cells in chronic lymphoid leukemia. N Engl J Med. 2011; 365: 725-733.

21. Kochenderfer JN, Dudley ME, Carpenter RO, Kassim SH, Rose JJ, Telford WG, Hakim FT, Halverson DC, Fowler DH, Hardy NM, Mato AR, Hickstein DD, GeaBanacloche JC, et al. Donor-derived CD19-targeted T cells cause regression of malignancy persisting after allogeneic hematopoietic stem cell transplantation. Blood. 2013; 122: 4129-4139.

22. Kochenderfer JN and Rosenberg SA. Treating B-cell cancer with $\mathrm{T}$ cells expressing anti-CD19 chimeric antigen receptors. Nat Rev Clin Oncol. 2013; 10: 267-276. 
23. Park JH and Brentjens RJ. Immunotherapies in CLL. Adv Exp Med Biol. 2013; 792: 241-257.

24. Ghorashian S, Pule M and Amrolia P. CD19 chimeric antigen receptor $\mathrm{T}$ cell therapy for haematological malignancies. Br J Haematol. 2015; 169: 463-478.

25. Levine BL. T lymphocyte engineering ex vivo for cancer and infectious disease. Expert Opin Biol Ther. 2008; 8: 475-489.

26. Kim JV, Latouche JB, Riviere I and Sadelain M. The ABCs of artificial antigen presentation. Nat Biotechnol. 2004; 22: 403-410.

27. Levine BL, Cotte J, Small CC, Carroll RG, Riley JL, Bernstein WB, Van Epps DE, Hardwick RA and June
$\mathrm{CH}$. Large-scale production of $\mathrm{CD} 4+\mathrm{T}$ cells from HIV-1-infected donors after CD3/CD28 costimulation. J Hematother. 1998; 7: 437-448.

28. Barrett DM, Singh N, Liu X, Jiang S, June CH, Grupp SA and Zhao Y. Relation of clinical culture method to T-cell memory status and efficacy in xenograft models of adoptive immunotherapy. Cytotherapy. 2014; 16: 619-630.

29. Xu Y, Zhang M, Ramos CA, Durett A, Liu E, Dakhova O, Liu H, Creighton CJ, Gee AP, Heslop HE, Rooney CM, Savoldo B and Dotti G. Closely related T-memory stem cells correlate with in vivo expansion of CAR.CD19-T cells and are preserved by IL-7 and IL-15. Blood. 2014; 123 : 3750-3759. 